for the first time possible through the enzymatic determination of galactose. By patients with liver disease the lowest level, 90 minutes after oral galactose administration is $30.3 \mathrm{mg}-\%$ galactose in blood. For the detection of a liver disease the 90 minute galactose value, after oral administration is sufficient.

Literatur. BAKx, C. J. A.: De bebaling van het galactose en glycosegehalte van het bloed na belasting van de leber met galactose. Ned. T. Geneesk. 90, 961 (1946). - BAUFR, R.: Über die Assimilation von Galaktose uns Milchzucker beim Gesunden und Kranken. Wien. med. Wschr, ä6, 20 (1906).- BRUECKNER, J.: Die Bestimmung der Cerebroside mit Hilfe des Pulfrich-Stufenphotometers. Hoppe-Seylers Z. physiol. Chem. 268 163 (1941); - ther den Gehalt des Blutes an Cerebrosiden. Hoppe-Seylers Z. physiol. Chem. 268, 251 (1941); - Die Unterscheidungsmöglichkeit kleiner Mengen von Cerebro-Glucosiden und Cerebro-Galactosiden. Hoppe-Seylers Z. physiol. Chem. 275, 73 (1942). - CoRLEY, R. C.: Factors in the metabolism of galactose. J. biol. Chem. 74, 1 (1927). - Deuler, J., and M. Dounororf: Metabolism of D-galactose in Pseudomonas saccharophila. J. biol. Chem. 227, 745 (1957). - Dovdoroff, M., C. R. Contropodrod, and S. Buras: Intern. Sympos. on Enzyme Chem. Tokio and Kyoto, Japan 2. 313 (1957). Fisher, H., R. G. HANSEN, and H. W. Norton: Quantitative determination of glucose and galactose. Analyt. Chem. 27, 857 (1955). - Harding, V. J., and G. A. Grant: Metabolism of galactose. J. biol. Chem. 99, $692(1932 / 33)$. - KAHLER, H. u. K. MachoLD: Utber das Verhalten des Blutzuckers nach Einnahme von Galaktose. Wien. klin. Wschr. 35, I, 414 (1922). 一 KA KHLFR, H.: Beiträge zur Leberfunktionspräfung. Med. Klin. 1925, 1295. - Ludwra, H.: Zar Technik und Bewertung der Galaktoseprobe. Z. klin. Med. 141, 758 (1942). PoLrak, F.: Die kombinierte Wassergalaktosebelastung als neue Leberfunktionsprobe. Z. klin. Med. 117, 476 (1931). Raymono, A. L., and J. B. Blanco: Blood sugar determination and separation of sugars with live yeast. J. biol. Chem. 79, 649 (1928). - REIss, E., u. W. JWHN: Alimentäre Galaktosurie bei Leberkrankheiten. Dtsch. Arch. klin. Med. 108, 187
(1912) - RoBINSOY, H. V. C., and J. C. RathruRN: Electrophoresis of free sugars in blood. Canad. J. Biochem. 35, 935 (1957). - Rommet, K., u. K. Grimmre: Oraler Galaktosetoleranztest. Med. Klin. 61, 44, 1735-1738 (1966); - Der orale Galaktosetoleranztest als Leberfunktionsprobe. Med. Klin. 62, 168-171 (1967). - RouBTrschek, R.: Alimentäre Galaktosurie bei experimenteller Phosphorvergiftung. Dtsch. Arch. klin. Med. 108, 225 (1912). — Scrmmt, E., u. F. W. Schmint: Enzym-Bestimmungen im Serum bei Lebererkrankungen. Enzymol. biol. clin. 3, 1 (1963). - SMrrH, M., and L. E. Tromas: Quantitative determination of glucose and galactose. Olin. Chem. 8, 289 (1962). - SøNDERGaARD, G.: Micro-method for determination of blood galactose by means of glucose oxidase (Notatin) and anthrone. Scand. J. elin. Lab. Invest. 10, 203 (1958). - StmarsTaM, T.: Peroral and intravenous galactose tests. Acta med. scand., Suppl. 177, 1-164 (1946). - Tyastrup, N., K. WrNkLeR, N. LUND, and H. C. Engrel : A elinical method for determination of plasma galactose in tolerance tests. Scand. J. clin. Lab. Invest. 6, 43 (1954). UexküLL, Te. v: Uber den diagnostischen Wert der Blutzuckerkurve nach peroraler Galaktosebelastung. Dtsch. med. Wsehr. 11, I, 415 (1939). - UexKÜLL, TH. v., u. H. H. WAGNER: Der Galaktosämietest als Leberfunktionsprobe, Münch. med. Wschr. 105, 1593 (1963). - WaLlentels, K., u. G. KuRz: Über die Spezifität der Galaktose-Dehydrogenase aus Psendomonas saccharophila und deren Anwendung als analytisches Hilfsmittel. Biochem. Z. 3855, 559 (1961). - WALLENFELs, K., and O. P. MALHotrA: Galactosidases. Advane. Carbohyd. Chem. 16, 239 (1961). - WATSON, D.: A new method for microanalysis of blood galactose. Ann. Biochem. 5, 260 (1963). - WoErner, H.: Toleranz gegen Galaktose bei direkter Einführung in den Pfortaderkreislauf. Dtsch. Arch. klin. Med, 110, 295 (1913).

Dr. K. Ronimer

Apotheker E. BERNT (C. F. Boehringer, Tutzing)

BSe F. SchMITZ

Dr. K. Grimmer

Universität Ulm

Zentrum für Innere Medizin.

$79 \mathrm{VIm}$, Steinhövelstr, 9

\title{
Serumproteinbindung von ACTH
}

\section{Untersuchungen mit Dextrangelfiltration und ${ }^{3} \mathrm{H}$-ACTH}

\author{
K. V. Werder, K. Schwarz and P. C. Soriba* \\ II. Medizinische Klinik der Universität München (Direktor: Prof. Dr. Dr. G. Bopeckrezi)
}

\section{Einleitung}

Untersuchungen über die Bindung von ACTH an Serumeiweißkörper liegen bisher nur spärlich vor. So konnten WoLF u. Mitarb. [1] zeigen, daB synthetisches, ${ }^{131} \mathrm{~J}$-markiertes Tetracosapeptid in der Radiopapierelektrophorese mit den Serumproteinen wanderte, wenn der Papierstreifen vorher mit nicht markiertem Corticotropin abgesättigt worden war. MELANI u. Mitarb. [2] fanden hingegen bei Dextrangelchromatographie von ${ }^{131} \mathrm{~J}$-markiertem Schweine-ACTH nur eine Bindung der radioaktiven Degradationsprodukte an das Serumprotein, wobei Angaben über die biologische Aktivität dieser "Degradationsprodukte" in dieser Arbeit fehlen [2]. - Biologische ACTH-Bestimmungen werden in unserem Labor seit einiger Zeit durchgeführt [3]. Da uns ${ }^{3} \mathrm{H}$-ACTH zur Verfügung stand, wurde mit Hilfe der Dextrangelfiltration, einer Technik, die wir zur Bestimmung der Proteinbindung von Schilddrüsenhormonen und Cortisol benützen $[4,5]$ die Frage der Bindung von ACTH an Serumproteine untersucht.

* Nit Unterstätzung der Dentschen Forschungsgemeinschaft und der Stiftung Volkswagenwerk (K. v. W.).

\section{Präparate und Methoden}

Als markiertes ACTH wurde ein katalytisch mit Tritium markiertes Tricosapeptid verwendet $\left({ }^{3} \mathrm{H}-\beta^{1-23}\right.$ Corticotropin23-amid-acetat, Farbwerke Hoechst AG), das in zwei spezifischen Aktivitäten vorlag: $212 \mu \mathrm{Ci} / \mathrm{mg}$ und $382 \mu \mathrm{Ci} / \mathrm{mg}$. Dabei entsprachen 1,0 mg Trockensubstanz ca. 100 E ACTH [6] Weiter wurde nicht markiertes $\beta^{1-23}$ Corticotropin-23-amid (Farbwerke Hoechst $A G$ ) und natives Schweine-ACTH (Cortrophine ${ }^{\circledR}$, Organon $\mathrm{GmbH}$ ) verwendet. Beiden Firmen sind wir zu großem Dank für ihre Unterstïtzung verpflichtet.

Die Dextrangelfiltration mit Sephadex G-25 fine warde als Säulenchromatographie mit Standardsäulen (i.D. $=0,8 \mathrm{~cm}$ ) durchgeführt. Sephadex wurde ca. $90 \mathrm{~min}$ in $0,01 \mathrm{M} \mathrm{Na}$ Phosphatpuffer pH 7,4 gequellt und darauf in eine vertikal stehende Säule, deren Ausfluß mit Glaswolle und einer Glasperlenschicht verschlossen war, gefïllt [4]. Das Gelbett wurde anschließend mit $3 \mathrm{ml} 5 \%$ iger Humanalbuminlösung (Behringwerke, $A G$ ) abgesättigt, da sich in Vorversuchen [7] gezeigt hatte, daß ohne vorherige Albuminabsättigung des Dextrangels das ACTH auch durch $0,1 \mathrm{~N} \mathrm{HCl}$ nicht vollständig eluiert werden konnte. Zur Elution dienten dann 0,01 M Na-Phosphatpuffer $\mathrm{pH} 7,4$ und $0,1 \mathrm{~N} \mathrm{HCl}$.

Die Messung der Radioaktivität erfolgte im Tri-Carb Liquid Szintillation-Spectrometer (Fa. Packard Instrument) mit $13,8 \%$ Ausbeute für Tritium in unserem System. Dafür wurden abhängig von der zu erwartenden Radioaktivität jeweils aliquote Teile der gesammelten Fraktionen $(3-5 \mathrm{ml})$ eines Versuches bis zur Trockene eingeengt, anschließend in $1,0 \mathrm{ml}$ 
Hyaminhydroxyd (Fa. Packard Instrument) gelöst und zu jeder Probe je $15 \mathrm{ml}$ Szintillationsflüssigkeit (Toluol-0,3\% PP0 - $0,06 \%$ POPOP) zugegeben. Die Quenchkorrektur erfolgte mit Hilfe der Internal Standard Methode ( ${ }^{3}$ H-Toluol). Protein wurde nach LowRY [8] bestimmt.

Die biologische Bestimmung der ACTH-Aktivität erfolgte mittels der von LrPscome und Nexson angegebenen Methode [9], die wir mit einigen Modifikationen anderer Autoren [10] in unserem Labor durehführen [3]. Dabei wird die ACTHAktivität anhand der Steigerung der Corticosteroninkretion im Nebennierenvenenblut der hypophysektomierten Ratte nach intraaortaler ACTH-Applikation bestimmt. Der Corticosterongehalt des Plasmas wurde nach der Methode von ZENKER und BERNSTEIN [11], modifiziert nach Preiffer u. Mitarb. [12] fluorimetrisch bestimmt.

Zur Reinigung des ${ }^{3} H$-ACTH wurde ebenfalls die Dextrangelfiltration herangezogen. In einem Vorversuch (Abb. 1) wurden $5 \mathrm{E}$ ungereinigtes ${ }^{3} \mathrm{H}$-ACTH in $0,5 \mathrm{ml} 0,9 \%$ igem $\mathrm{NaCl}$ über eine albumingesättigte Säule $(h=15 \mathrm{~cm})$ gegeben. Anschließend wurde mit 0,01 M Na-Phosphatpuffer pH 7,4 und darauf mit $0,1 \mathrm{~N} \mathrm{HCl}$ eluiert, $0,5 \mathrm{ml}$ jeder Fraktion $(5 \mathrm{ml})$ wurden jeweils zur Flüssigkeitsszintillationszählung aufbereitet. In den Fraktionen 2 und 9 wurde dazu die biologische ACTH-Aktivitä bestimmt. Wie die Abbildung zeigt befindet sich die biologische ACTH-Aktivität allein im Säureeluat. Die unspezifische Radioaktivität ohne biologische ACTH-Aktivität wurde durch den Na-Phosphatpuffer pH 7,4 eluiert. Die Rechromatographie nach Neatralisation [7] der beiden Radioaktivitätsgipfel nach dem gleichen Verfahren ergab jeweils nur den einen entsprechenden homogenen Gipfel ( $\mathrm{Abb}$. $2 a$ und $\mathrm{b}$ ). Für die folgenden Versuche wurde nach diesem Verfahren gereinigtes ${ }^{3} \mathrm{H}$-ACTH verwendet. Dabei betrug die unspezifische Radioaktivität ohne biologische ACTH-Aktivität nach Reinigung 3-4\% des einmal chromatographierten ${ }^{3} \mathrm{H}$-ACTH $(\mathrm{Abb} .2 \mathrm{~b})$.

\section{Ergebnisse}

In Vorversuchen [7, 13] mit nichtmarkiertem synthetischem ACTH und nativem Schweine-ACTH wurde deut́lich, daß Serumproteine und Dextrangel kompetitiv biologisch aktives ACTH zu binden vermögen. Durch die radioaktive Markierung des Hormons wurde es möglich, diese Untersuchungen quantitativ durchzuführen. Dazu wurden $200 \mathrm{mE}$ chromatographisch gereinigtes, mit $1,0 \mathrm{~N} \mathrm{KOH}$ reneutralisiertes (ca. pH 7) ${ }^{3} \mathrm{H}$-ACTH mit $2,0 \mathrm{ml}$ Normalserum und $1,2 \mathrm{ml} 0,9 \%$ igem $\mathrm{NaCl}$ in einem Gesamtvolumen von $4,0 \mathrm{ml}(0,0015 \mathrm{M}$ Na-Phosphatpuffer $\mathrm{pH} 7,4) 3 \mathrm{~min}$ bei $+37^{\circ}$ inkubiert. $2 \mathrm{ml}$ des Inkubationsgemisches wurden auf eine albumingesättigte Dextrangelsäule gegeben. Die Elution erfolgte zuerst mit $0,01 \mathrm{M} \mathrm{Na}$ Phosphatpuffer $\mathrm{pH} 7,4$ und anschließend mit $0,1 \mathrm{~N}$ $\mathrm{HCl}$ (Abb. 3). Die Abbildung zeigt deutlich, daß der gröBere Teil des ${ }^{3} H-A C T H$ zusammen mit den Serum. proteinen eluiert wurde; der sog. freie, d.h. dextrangelgebundene Teil des ${ }^{3} \mathrm{H}$-ACTH warde durch die Salzsäure eluiert. Die Gesamtausbeute an Radioaktivität betrug $95 \pm 7,6 \%(\bar{x} \pm s, N=55$ Säulen $)$.

Mit der Proteinbindung tritt gleichzeitig eine erhebliche Inaktivierung des synthetischen Tricosapeptides ein, wie die geringe biologische Aktivität (Abb. 3) des proteingebundenen ${ }^{3} \mathrm{H}$-ACTH zeigt [14].

Analoge Versuche mit nativem Schweine-AOTH und biologischer Bestimmung der ACTH-Aktivität im Eluat zeigten demgegenüber (Abb.4) keine wesentliche Inaktivierung der biologischen Aktivität durch Inkubation mit Serumprotein $[7,14]$, und demonstrieren die Serumproteinbindung von natürlichem ACTH. - Wurden $100 \mathrm{mE}{ }^{3} \mathrm{H}$-ACTH mit verschiedenen Serummengen $(0,1-2,0 \mathrm{ml}$ Normalserum) inkubiert und die Inkubationsgemische anschließend auf Dextrangelsäulen konstanter Höhe gegeben und in üblicher Weise zuerst mit $0,01 \mathrm{M}$ Na-Phosphatpuffer

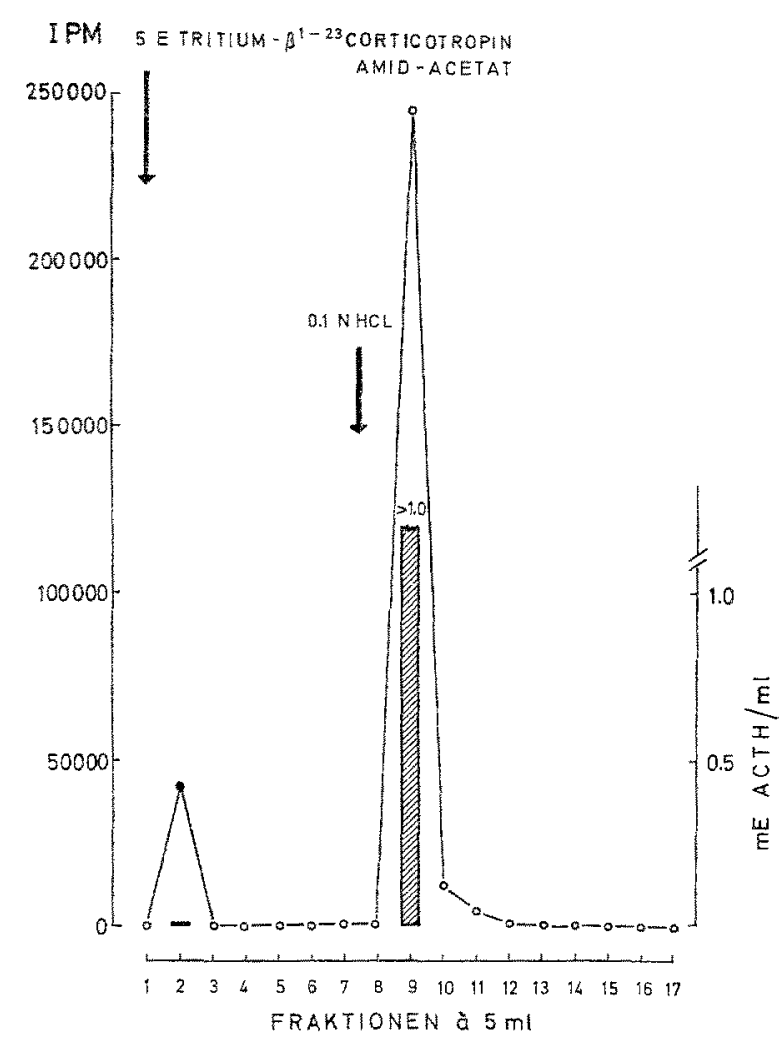

$\mathrm{A} b$ b. 1. Dextrangelfiltration von $5 \mathrm{E}$ ungereinigtem ${ }^{3} \mathrm{H}$-ACTH. IPM (o__ o), biologische ACTH-Aktivität (Säulen)

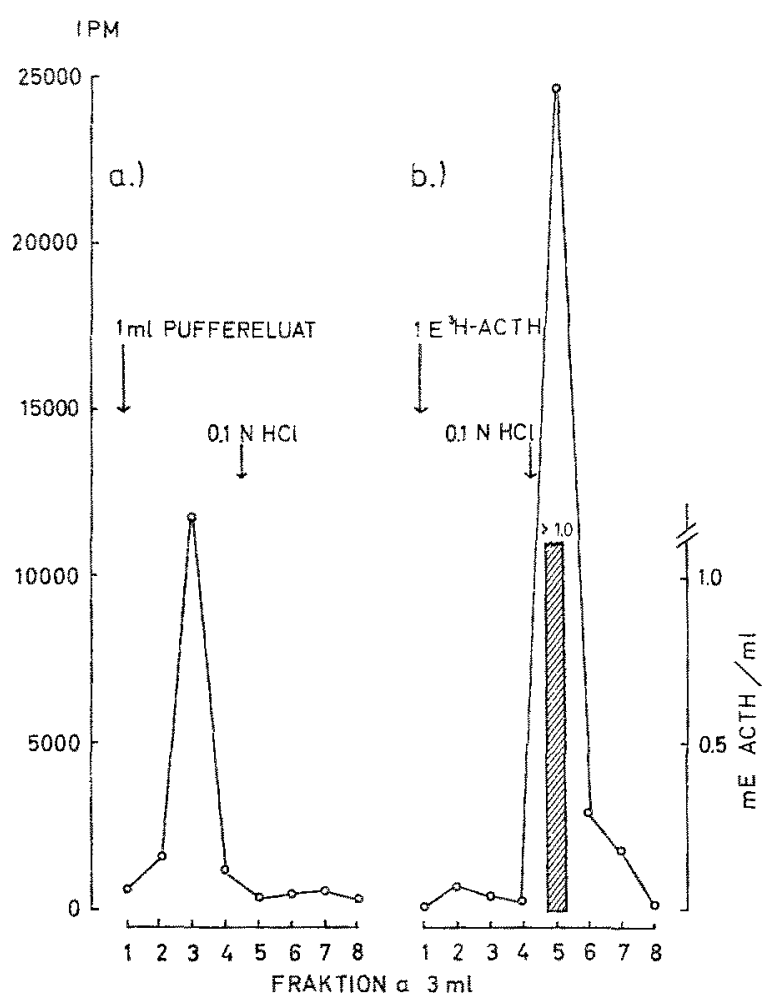

Abb. 2. a Rechromatographie von $1 \mathrm{ml}$ Pufferelnat von ungereinigtem ${ }^{3} \mathrm{H}$-ACTH $(=$ unspezifische Radioaktivität ohne biologisehe Aktivität). $\mathrm{b}$ Rechromatographie von $1 \mathrm{ml}=1 \mathrm{E}$ ${ }^{3} \mathrm{H}$-ACTH. IPM (o - o) in $0,5 \mathrm{ml}$ jeder Fraktion, biologische ACTH-Aktivitat (Süulen)

$\mathrm{pH} \mathrm{7,4}$ und darauf mit $0,1 \mathrm{~N} \mathrm{HCl}$ eluiert, so nimmt der proteingebundene Anteil des ${ }^{3} \mathrm{H}$-ACTH mit steigenden Serummengen im Inkubationsgemisch zu 
IPM

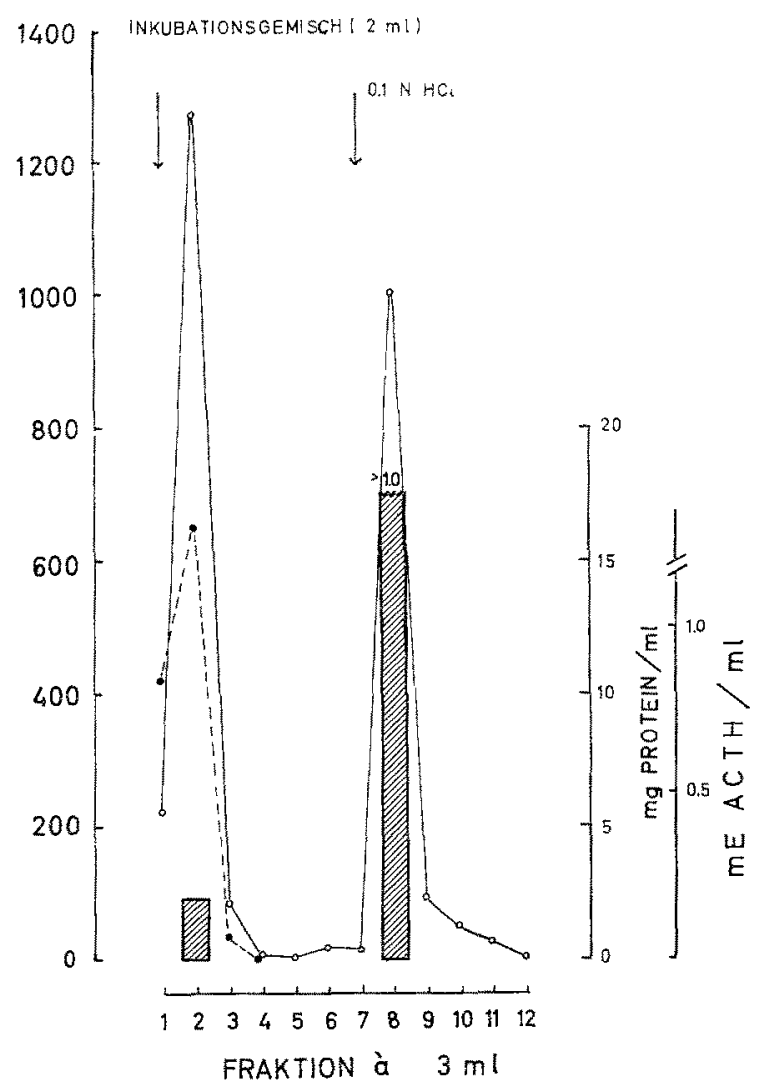

Abb. 3. Dextrangelfiltration von 2,0 $\mathrm{ml}$ Inkubationsgemiseh (100 mE ${ }^{3} \mathrm{H}$-ACTH $+1,0 \mathrm{ml}$ Serum). Säulenhöhe: $8,5 \mathrm{~cm}$, IPM (०- 0 ) in $0,5 \mathrm{ml}$ jeder Fraktion, Protein $(\bullet-\bullet)$, biologische ACTH-Aktivität (Säulen)

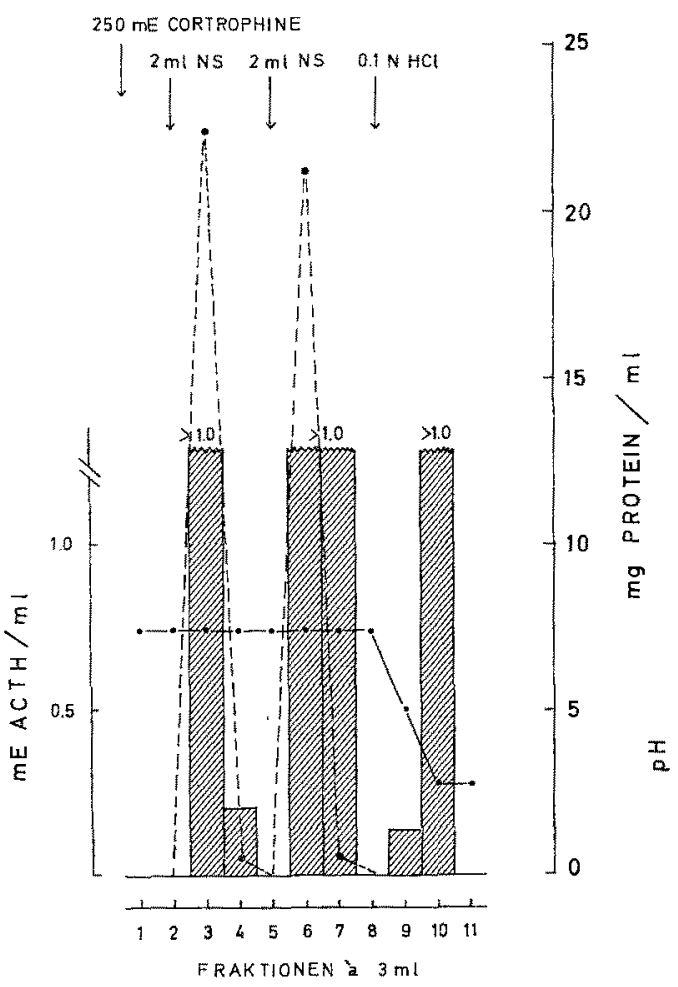

Abb. 4. Dextrangelfiltration von $250 \mathrm{mE}$ Schweine-ACTH (Cortrophine(B). Zweimaliges Waschen der Säule mit Normalserum. Anschließend Elution mit $0,1 \mathrm{~N}$ HCl. Biologische

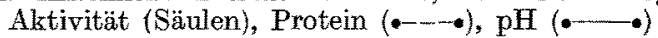

(Abb. 5). Umgekehrt nimmt der sog. freie, d.h. dextrangelgebundene Anteil des Tricosapeptids mit $\mathrm{Zu}$. nahme der Serummengen im Inkubationsgemisch ab. Wird der Anteil des proteingebundenen ${ }^{3} \mathrm{H}$-ACTH in $\%$ der Gesamtradioaktivität angegeben, so wird bei zunehmender Serumproteinmenge (bis $4,0 \mathrm{ml}$ ) ein Wert von $73 \%$ proteingebundenem Tricosapeptid erreicht.

Diese kompetitive Bindung des ${ }^{3} \mathrm{H}$-ACTH dureh Serumproteine und Dextrangel wurde durch folgende Versuche bestätigt. $2 \mathrm{ml}$ eines Inkubationsgemisches (vgl. Text zu Abb. 3) wurden auf Dextrangelsäulen

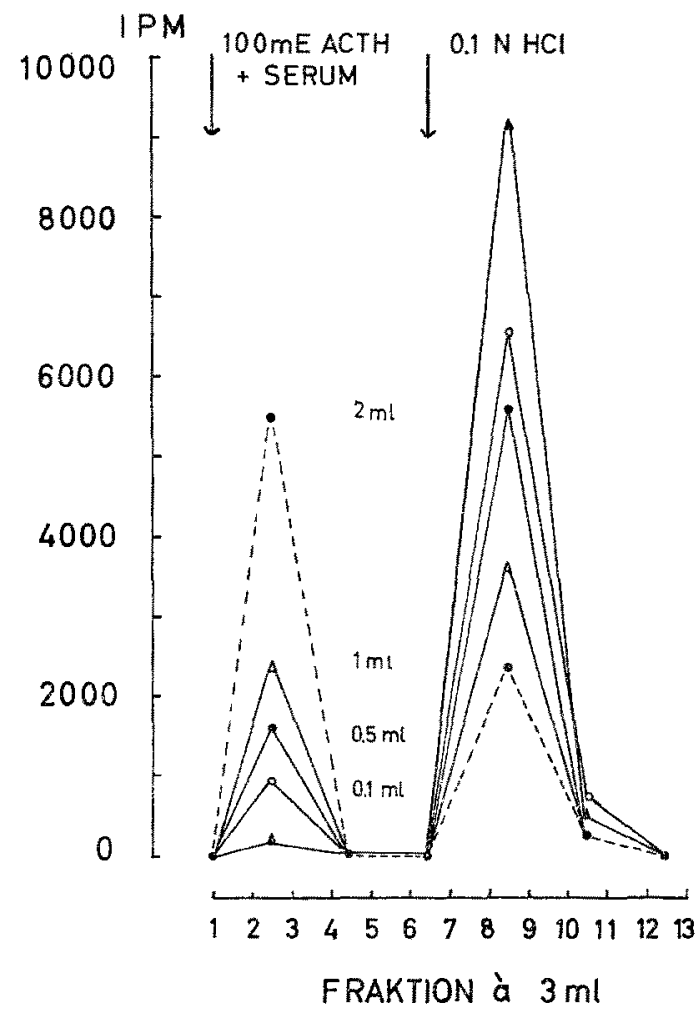

Abb. 5. Dextrangelfiltration von $100 \mathrm{mE}$ gereinigtem ${ }^{3} \mathrm{H}$ ACTH, inkubiert mit verschiedenen Serummengen. Säulenhöhe konstant: $15 \mathrm{~cm}$, Serummenge: $2 \mathrm{ml}(\bullet-\cdots), 1 \mathrm{ml}$

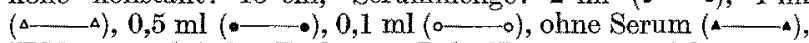
IPM in $1 \mathrm{ml}$ jeder Fraktion. Jede Kurve entspricht einem Versuch

verschiedener Höhe $(h=1,2-18,0 \mathrm{~cm}$, i. D. $=0,8 \mathrm{~cm})$ gegeben. In üblicher Weise wurde zuerst mit Puffer und anschließend mit 0,1 N HCl eluiert. Dabei ergab sich, daB mit Abnahme bzw. Zunahme der Säulenhöhe der proteingebundene Anteil des ${ }^{3} \mathrm{H}$-ACTH (Puffereluat) zunahm, bzw. abnahm. Die Abb. 6 zeigt die Verminderung des proteingebundenen ${ }^{3} \mathrm{H}$-ACTH in \% der eluierten Gesamtaktivität bei Steigerung der Säulenhöhe. Extrapolation auf $0 \mathrm{~cm}$ Säulenhöhe führt ebenfalls zu einem Wert von $70-80 \%$ proteingebundenem Tricosapeptid. Wurde an Stelle von Serumprotein mit $5 \%$ Humanalbuminlösung inkubiert, so zeigte sich bei der anschließenden Dextrangelfiltration eine Bindung des ${ }^{3} H-A C T H$ an Albumine (Abb. 7), die der Bindung an Serumproteine (vgl. Abb.6) etwa entsprach.

Um das Verhalten von endogenem $A C T H$ bei der Dextrangelfiltration zu klären, wurden 2,0 ml Plasma einer adrenalektomierten Patientin mit hohem ACTHPlasmaspiegel (Verdacht auf R-Zellen Adenom [15] des Hypophysenvorderlappens) auf eine Dextrangel- 
säule gegeben und in üblicher Weise eluiert (Abb. 8). Wie die Abbildung zeigt, wurde in der ersten Fraktion (Puffereluat) biologisch wirksames ACTH gefunden, welches den proteingebundenen Anteil darstellt. Durch die Salzsäure wurde anschließend das dextrangelge. bundene bzw. sog. freie ACTH eluiert. Obwohl wegen der Ungenauigkeit der biologischen ACTH-Bestimmungen [3] keine Rückschlüsse hinsichtlich der quantitativen Verteilung von proteingebundenem und sog. freiem ACTH aus diesem Versuch gezogen werden können, ist doch auch hier die kompetitive Bindung von in diesem Falle endogenem ACTH durch Serumprotein und Dextrangel deutlich.

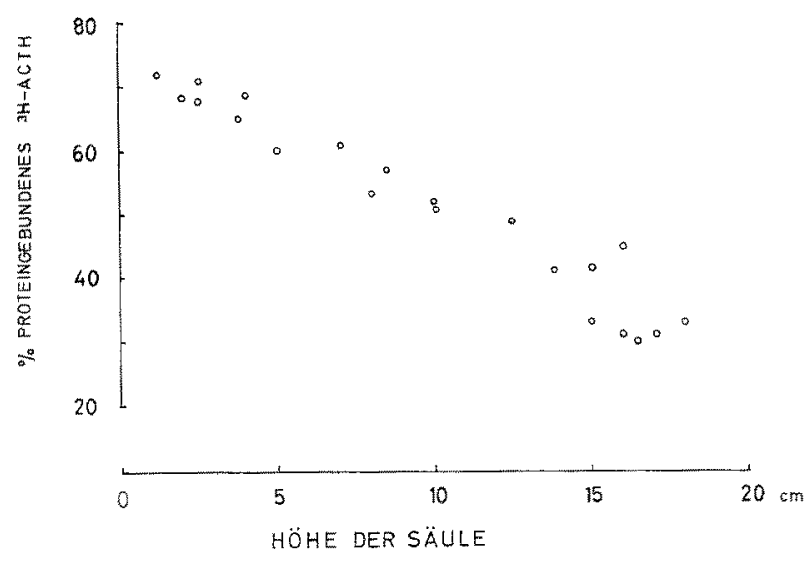

Abb. 6. Einfluß der Höhe der Sephadfex-G-25-Säule auf den proteingebundenen ${ }^{3} \mathrm{H}$-ACTH-Anteil (aufgetragen: $100 \mathrm{mE}$ mit $1 \mathrm{ml}$ Serum in $2 \mathrm{ml}$ Inkubationsgemisch)

\section{Diskussion}

Die für Schilddrüsenhormone und Cortisol $[4,5]$ schon zu diagnostischen Zwecken ausgenutzte Bestimmung der Proteinbindung dieser Hormone ist auch für einige Peptidhormone beschrieben worden [16, 17]. Uber ACTH $[1,2]$ lagen bisher widersprüchliche Ergebnisse vor (s. Finleitung).

Wir fanden bei der Dextrangelfiltration eine starke Bindung des Tritium markierten $\beta^{1-23}$ Corticotropins an das Dextrangel, die durch Elution mit $0,1 \mathrm{~N} \mathrm{HCl}$ allein nicht zu lösen war (Vorversuche). Erst nachdem. wir die Säulen mit 5\% iger Albuminlösung abgesättigt hatten, konnten wir durch Serumproteine und $0,1 \mathrm{~N}$ $\mathrm{HCl}$ oder Salzsäure allein das ${ }^{3} \mathrm{H}-\mathrm{ACTH}$ vollständig von der Dextrangelsäule eluieren [7, 18]. Dabei zeigte sich eine eindeutige Kompetition von Serum und Dextrangel bezüglich der ACTH-Bindung. Durch Veränderung der Dextrangel- bzw. Serummenge ergab sich mittels Extrapolation eine Bindung von $70-80 \%$ des ACTH an Serumproteine bei allerdings extrem unphysiologisch hohen ACTH-Konzentrationen. Der Einwand, daß es sich bei dieser Versuchsanordnung um eine Verdrängung von ACTH aus der Dextrangelsäule durch Proteine und nicht um eine echte Bindung an Proteine handelt, muß genauer analysiert werden.

1. Theoretisch ist es denkbar, daß bei der Albuminsättigung nur eine sehr geringe Albuminmenge dextrangelgebunden wird, welche bei der Säulenchromatographie von ACTH-Lösungen ohne Serumproteinzusatz durch die möglicherweise größere ACTH-Peptidmenge teilweise von der Säule ,verdrängt" wird. Folgt dem eine Elution mit überschüssigem Albumin, bzw. Serumprotein (7-280 mg Protein), so "verdrängt" dieses Protein seinerseits das ACTH, welches somit nicht protein. gebunden eluiert wärde. Gegen diese Möglichkeit spricht die

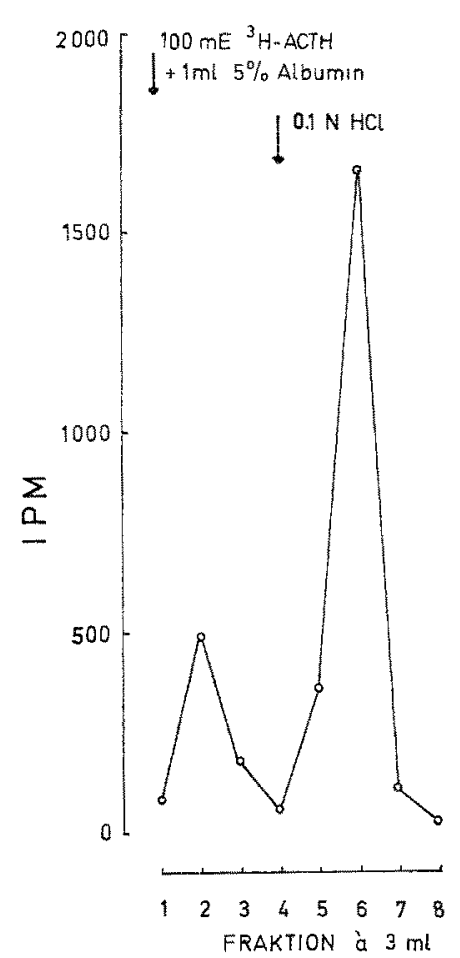

Abb. 7. ${ }^{3}$ H-ACTH-Bindung an Albumin. Inkubation von $100 \mathrm{mE}{ }^{3} \mathrm{H}-\mathrm{ACTH}$ mit $1 \mathrm{ml} 5 \%$ igem Humanalbumin $(\Sigma V=$ $2 \mathrm{ml}$, vgl. Text zu Abb. 3) Dextrangelfiltration (Säulenhöe = $16 \mathrm{~cm}$ ). Elution mit $0,01 \mathrm{M}$ Na-Phosphatpuffer $\mathrm{pH} 7,4$, dann mit $0,1 \mathrm{~N} \mathrm{HCl}$. IPM $\left(0_{-} \circ\right)$ in $0,5 \mathrm{ml}$ jeder Fraktion

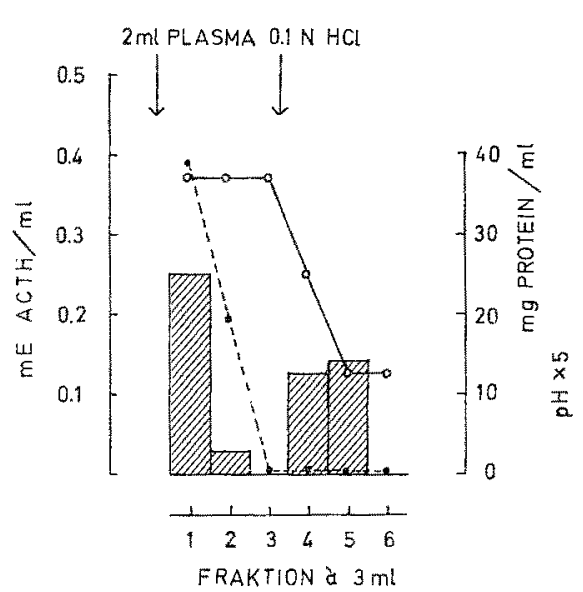

Abb. 8. Dextrangelfiltration von $2 \mathrm{ml}$ Patientenplasma (M.K.) mit erhöhter endogener ACTH-Aktivität. Säulenhöhe: $7 \mathrm{~cm}$, biologische AOTH-Alktivität (Säulen) Serumprotein (•---.), $\mathrm{pH}(\mathrm{o}-\mathrm{o})$

Uberlegung, daB ein ACTH (1 $\mu \mathrm{g})$, welches Albumin von dex gesä,ttigten Säule, verdrängen" sollte, genauso fest dextrangelgebunden sein müBte, wie ACTH, welches auf nicht mit Albumin gesättigte Säulen gegeben wurde. Dieses ACTH wäre nicht vollständig durch $0,1 \mathrm{~N}$ HCl-Zugabe, wie gezeigt [7], von der Säule zu eluieren. Da $0,1 \mathrm{~N}$ HCl jedoch das ACTH von dex albumingesättigten Säule nahezu vollständig eluierte, scheidet diese Möglichkeit aus.

2. Es ist darüber hinaus unwahrscheinlich, da.8 $1 \mu g$ ACTH in proteinfreier Lösung das Albumin aus mit $150 \mathrm{mg}$ albumin. gesättigten Säulen so quantitativ ,verduängen" kann, daß nicht doch ein gewisser Anteil des ACTH die Säule passiert. Wix fanden dagegen bei der Chromatographie von ${ }^{3} \mathrm{H}-\mathrm{ACTH}$ ohne Serumzusatz auf albumingesättigten Säulen in den ersten Fraktionen nur $3-4 \%$ unspezifisehe Radioaktivität ohne biologische Aktivität.

3. Wir müssen daher diskutieren, daß das Dextrangel dem ACTH zwei Bindungsmöglichkeiten bietet. 
Der eine "Acceptor" bindet das ACTH so, daß auch durch $0,1 \mathrm{~N}$ HCl diese Bindung nicht gelöst werden kann [7], wohingegen der zweite ,Acceptor" dass ACTH reversibel bindet. Ist der erste ,Acceptor" durch Albumin abgesättigt, so wird das ACTH nur noch labil an den zweiten gebunden und kann durch Serum und/oder Salzsäure vollständig eluiert werden. Für den zweiten ,Acceptor" des Dextrangels gelten wieder die unter 2, ausgeführten Überlegungen.

Dabei gilt für die zwei rein hypothetischen ,Acceptoren" die Uberlegung, daß die physikalisch-chemische Natur des Adsorptionsvorganges vermutlich komplex, d.h. zusammengesetzt aus der Wirkung Coulomb. scher-, Van der Waalsscher Kräfte u.a. ist [19]. Die physikalisch-chemischen Grundlagen der Adsorption von ACTH an Dextrangel wurden hier nicht untersucht. Das erwähnte Modell soll nur dazu dienen, die erhaltenen Ergebnisse zu interpretieren. Dabei soll noch einmal hervorgehoben werden, daß das Dextrangel bei der Chromatographie von ACTH nicht als Molekülsieb wirkt, sondern das ACTH-Peptid bindet. Die Affinität von Tyrosin enthaltenden Peptiden [19] und Tyrosinderivaten, z.B. Trijodthyronin [4] sowie anderen Verbindungen, z,B. Corticosteroiden [5] zu Dextrangel (Sephadex G-25) ist bekannt. Die erheblichen Diskrepanzen zu den Befunden von Merani [2] sind u.a. möglicherweise durch eine Änderung der physikalisch-chemischen ACTH-Dextrangelbeziehung bei Jodierung des ACTH-Moleküls an den Tyrosinen zu erklären. - Wir müssen in diesem Zusammenhang auch offen lassen, welche Rolle die Tatsache, daß Albumine die Dextrangelsäule mit dem AusschluBvolumen passieren (bei Sephadex G-25 werden Moleküle mit einem Molekulargewicht von mehr als ca. 5000 ausgeschlossen) für die Änderung der ACTH-Bindung in albuminisierten Säulen spielt. ACTH könnte nach seinem Molekulargewicht (ca. 2500-4500, je nach Länge des ACTH-Peptids) in das innere Gelvolumen eindringen. Gegen die Möglichkeit, daß die stabilere (s.o.) ACTH-Bindung im inneren Gelbettvolumen stattfindet und die Albumine das ACTH am Eindringen in das innere Gelbettvolumen hindern, spricht die Beobachtung, daß Serumproteine, die nach dem ACTH auf eine nicht mit Albumin gesättigte Säule gegeben werden, die stabile ACTH-Bindung an Dextrangel so veränderten, daß eine vollständige Elution durch Säure wieder möglich wurde [7]. Daß es sich wirklich um eine echte Bindung des ACTH an Serumprotein und nicht nur um eine Verdrängung vom Dextrangel handelt, konnte auch durch Saccharosedichtegradientenzentrifugation von $\beta^{1-23}$ Corticotropin bewiesen werden [20].

Zusammenfassung. ${ }^{5} \mathrm{H}-\beta^{1-23}$.Corticotropin wurde an Dextrangel (Sephadex G-25) gebunden und konnte durch Serumproteine, Albumin oder 0,1 N HCl eluiert werden. Mittels Dextrangelfiltration wurde gefunden, da ${ }^{3}$ H-ACTH kompetitiv an Serumproteine (Albumin) und Dextrangel gebunden wurde. Auch für natürliches Schweine-ACTH und endogenes ACTH in Patientenplasma (Adrenalektomie) wurde mittels biologischer ACTH-Bestimmung die Bindung von ACTH an Proteine bestätigt.

Summary. ${ }^{3} \mathrm{H}-\beta^{\mathbf{1}-23}$ corticotropin was bound to dextran gel (sephadex $G-25$ ) and was eluted by either serum proteins, albumin or $0.1 \mathrm{~N} \mathrm{HCl}$. Competitive binding of ${ }^{3} \mathrm{H}$-ACTH to serum proteins (albumin) and dextran gel was shown by dextran gel filtration. Likewise natural ACTH (pig) and endogenous ACTH from plasma of an adrenalectomized patient were shown to be partly protein bound using biological ACTH-assay.

Frl. F. SegdiMaIER und Frl. S. v. GüNTHER danken wir für ihre ausgezeichnete technische Assistenz. Auszungweise vorgetragen [18] beim VI. Acta Endocrinologica Congress, Helsinki, 1967.

Literatur. 1. Wolf, R. L., M. MandLOWITZ, L. J. SOFFER, J. RoBoz, and S. E. GITLow: Metabolism of corticotropin in man. Proc. Soc. exp. Biol. (N.Y.) 119, 244 (1965). - - 2. MELANT,

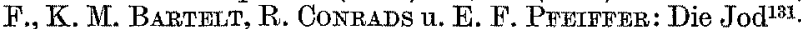
Markierung von Insulin, ACTH und STH mit hoher spezifiseher Aktivität zur Anwendung in der radioimmunologischen $\mathrm{Me}-$ thode. Z. klin. Chem. 4, 189 (1966). - 3. SCRIBA, P. C., R. Hacker, P. Dieterlee, F. Kudge, W. Hochmedser u. K. SCHWARZ: ACTH-Bestimmungen im Plasma aus dem Bulbus cranialis venae jugularis. Klin. Wschr. 44, 1393 (1966). 4. Soriba, P. C., R. Lantdgraf, H. G. Hetngze u. K. Schwarz: Bestimmung der Bindung von Trijodthyronin an Serumproteine mittels Dextrangel-Filtration. Klin. Wschr. 44, 69 (1966). - 5. MÜller-Bardorfi, M., W. HochHeUSter, K. Sorwarz, and P. C. SortBa: Effect of ACTH-infusion tests on Cortisol-4-C-14 binding to plasma proteins. Acta endocr. (Kbh.), Suppl. 119, 180 (1967). - 6. VoGEL, H. G.: Evaluation of synthetie peptides with ACTH-activity. Acta endocr. (Kbh.), Suppl. 100, 34 (1965). - 7. WERDER, K. v.: Serumproteinbindung von Adrenocorticotropin. Ein Beitrag zum Problem der Bestimmung von ACTH-Plasmaspiegeln. Diss. Univ. München 1968. - 8. Lowry, O. R., N. J. Rosebrovgh, A. L. FaRR, and R.V.RANDaLL: Protein measurement with the Folinphenol-reagent. J. biol. Chem. 193, 265 (1951). - 9. LiPscomr, H. S., and D. H. NELSON: A sensitive biologic assay for ACTH. Endocrinology 71, 13 (1962). - 10. GIRARD, F., and V. K. VANCE: Modification of the Lipscomb-Nelson bioassay for ACTH. Endocrinology 71, 325 (1962). - 11. ZENK KR, N., and D. E. BERNSTEIN : The estimation of small amounts of corticosterone in rat plasma. J. biol. Chem. 231, 695 (1958). 12. PFeiffer, E. F, W. E. Vaubei, K. Remiene, D. Berg u. H. DitschunetT: ACTH.Bestimmung mittels Messung des Plasma Corticosterons der mit Dexamethason hypophysenblockierten Ratte. Klin. Wschr. 38, 980 (1960). - 13. KuUGE, F.: Zur biologischen Bestimmung von ACTH-Plasmaspiegeln: Verteilung von endogenem ACTH im Körper und Bindung an Serumproteine. Diss. Univ. München 1967. - 14. ImuRA, H., H. Matsuyama, S. Matsukura, T. Mtyake, and M. Furase: Stability of ACTH preparations in human plasma incubated in vitro. Endocrinology 80, $599(1967)$. - 15. KRACHT, J., H. D. Zrmmermann u. U. Hachmetster: Immunhistologischer Nachweis in einem R-Zellen Adenom des Hypophysenvorderlappens bei M. Cushing. Virchows Arch. path. Anat. 340, 270 (1966). - 16. ANTONIAdEs, H. N., A. M. HuBER, B. R. BoSHELL, C. A. SARAVIS, and S. N. GERSHOFF: Studies on the state of insulin in blood: Properties of circulating "free" and "bound" insulin. Endocrinology 76, 709 (1965). - 17. MAC MruLAN, D. R., J. M. Schmid, and S. A. EASH: Studies on the heterogeneity and serum binding of human growth hormone. J. clin. Endocr. 27, 1090 (1967). - 18. WERDER, K. v., F. KLUGE, K. SChwARZ, and P. C. SCRTBA: Determination of serum protein binding of $3 \mathrm{H}-\mathrm{ACTH}$ using dextran gel filtration and density gradient centrifugation. Acta endocr. (Kbh.), Suppl. 119, 182 (1967). - 19. DetERMranN, H.: Gelchromatographie. Berlin-Heidelberg-New York: Springer 1967. 20. Werder, K. y., F. Kluqe, K. Sohwarz u. P. C. Scriba Serumproteinbindung von ACTH. II. Untersuchungen mit Dichtegradienten-Zentrifugation und 3H-ACTH. (Erscheint in Klin. Wschr. 1968.)

Dr. K. v. WERDER

Prof. Dr. K. SoHWARZ

Priv.-Doz. Dr. P. C. Scriba

II. Medizinisehe Klinik der Univ. 8 München 15, Ziemssenstr. 1 